\title{
Pain relief in a patient with massive sino-nasal tumor
}

\author{
Ali Usman, Fahad Rafiq Butt, Romana Shaida Durrani, Ateeq Ur Rehman Ghafoor
}

Department of Anesthesia \& Pain Medicine, Shaukat Khanum Memorial Hospital \& Research Centre, Lahore, Pakistan.

Correspondence: Dr. Romana S. Durrani; E-mail: romanadurrani@gmail.com

\section{Abstract}

Maxillofacial neoplasms, though relatively rare, are potentially devastating tumors, that may require medical or surgical intervention. Among all the head and neck tumors, sino-nasal tumors make up 3\%. Such tumors can be very vascular as well as extremely painful. Conventional analgesic treatment in a cascading manner usually proves to be inadequate and unsatisfactory. Interventional pain management offers promising long term pain relief. Sphenopalatine ganglion block can be offered to such patients for pain relief. Here we present a case report of a patient with painful large sino-nasal tumor for which spheno-palatine ganglion (SPG) block was performed with successful pain relief.

Key words: Pain; Pain management; Tumor; Spheno-palatine ganglion

Abbreviations: SPG - spheno-palatine ganglion; NSAIDs - non-steroidal anti-inflammatory drugs; PACU - post anesthesia care unit; RFA - radiofrequency ablation; PRF- pulsed radiofrequency

Citation: Usman A, Butt FR, Durrani RS, Ghafoor AR. Pain relief in a patient with massive sino-nasal tumor. Anaesth. Pain Intensive Care 2020;24(4):457-459.

Received: 22 June 2020, Reviewed: 24 June 2020, Accepted: 29 June 2020

\section{Introduction}

Maxillofacial cancers, though relatively rare, are potentially devastating disease that may require medical or surgical intervention. Among all the head and neck tumors, sino-nasal tumors comprise 3\%., Such tumors can be very vascular as well as extremely painful. Sphenopalatine ganglion block can be offered to such patient for pain relief. Here we present a case report of a patient with painful large sino-nasal tumor for which spheno-palatine ganglion (SPG) block was done with successful pain relief. This patient had been on multiple analgesics, oral and even parenteral, but her pain could not be relieved. SPG successfully made her comfortable and pain free. The case report is presented to emphasize the importance of interventional pain procedures and the need to keep this option in mind, when conventional methods fail.

\section{Case Report}

A young, 18 years old female, from Afghanistan presented in our Pain Medicine Clinic with large painful swelling over the face for four months. It progressively had increased in size and was associated with weight loss. Other symptoms included frequent episodes of fever, headache and odynophagia. The patient presented histopathology report that showed benign epithelial tumor. On examination there was huge tumor mass occupying nose completely and middle face, deviating mouth inferiorly. Intraorally it was compressing the hard palate. There was no cervical or any other lymphadenopathy.

Repeat histopathology in our hospital showed it to be sinonasal tumor of salivary gland origin (T4 N0). MRI face and neck demonstrated a large heterogeneously enhancing left sided sino-nasal mass likely arising from the left maxillary antrum. It measured 94 x 97 $\mathrm{mm}$ in maximum coronal dimensions and showed significant surrounding mass effect on the nasal septum, ipsilateral zygomatic bone and infero-medial orbital wall (Figures $1 \& 2$ ). She had palliative quad shot radiotherapy. Repeat CT scan of head after third shot showed no improvement and inoperability. Further application of radiotherapy was stopped. 
Pain at the site of tumor became excruciating, not relieved with regular dose of analgesics that included mild opioid, NSAIDs and neuropathic drugs. The next option was pain intervention. Sphenopalatine block was explained to the patient with all the possible side effects and failure possibility. The patient decided to proceed with intervention. Patient was booked for operating room for SPG chemical ablation with fluoroscopic guidance under sedation.
On procedure day patient came with $6 \mathrm{~h}$ fasting. After securing intravenous access, patient was positioned supine and sedation was induced with target controlled infusion (TCI) of propofol. The patient continued breathing spontaneously and no airway device was used. Under fluoroscopic guidance after injecting $2 \%$ lignocaine in skin, Quincke spinal needle 22G was advanced with lateral infratemporal approach. Needle tip position was confirmed with radio opaque dye and
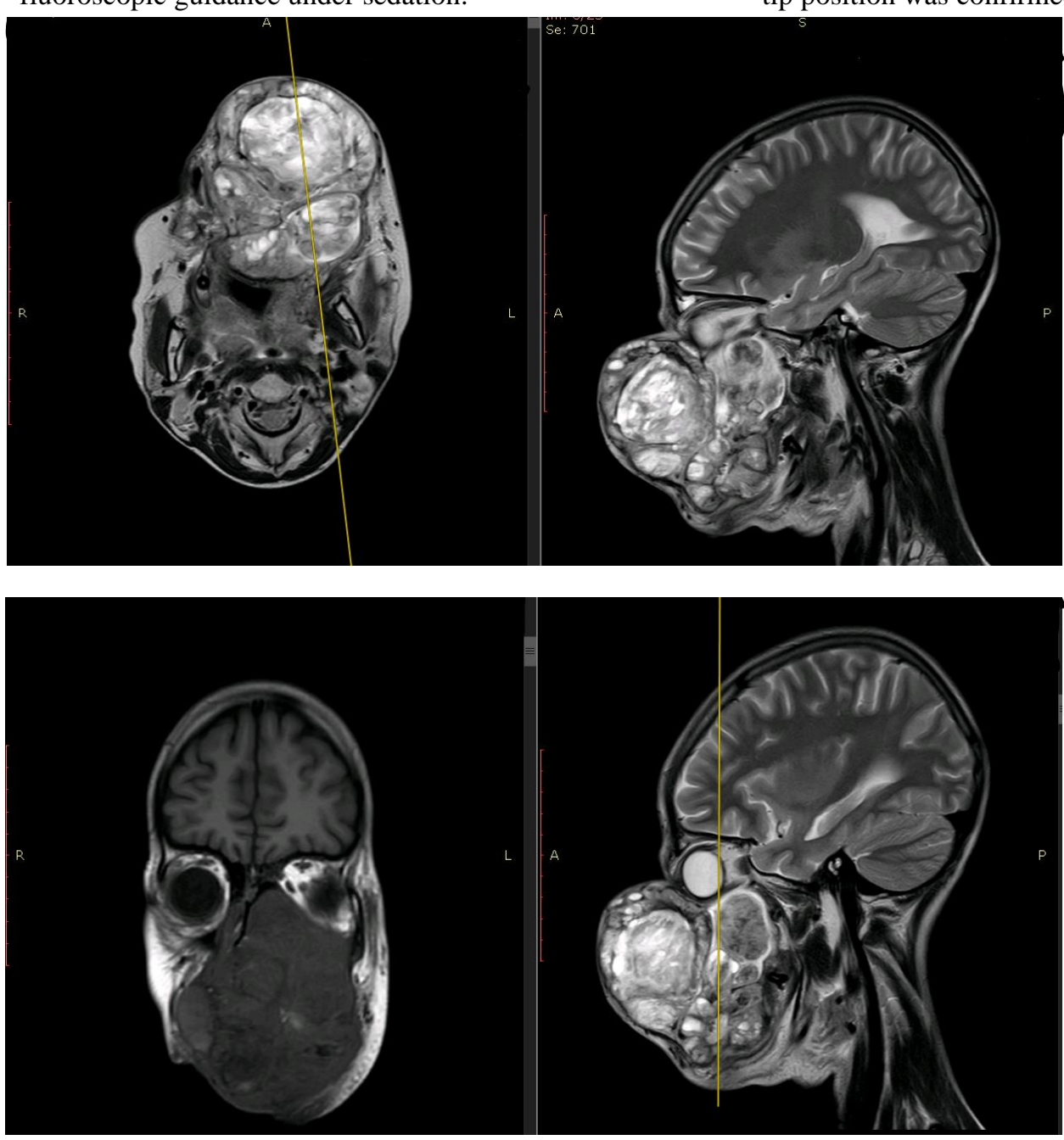

then chemical ablation was performed with $1 \mathrm{ml}$ of $6 \%$ phenol. After the procedure she was observed in post anesthesia care unit (PACU) for $30 \mathrm{~min}$. On her return to clinic for follow up after a month, there was no pain and she was off all pain medications. She was referred back to her primary team for further possibilities of palliation.

Unfortunately, the procedure images were lost due to connection issues of $\mathrm{C}$ arm image intensifier to the central system and available photos on cell phone are of poor resolution.

Figure 1 \& 2: Axial, sagittal and coronal images of T1 and T2 sequence showing heterogeneous large abnormal intensity lesion involving the maxillary sinuses as well as almost all the facial structures mainly on the left side. The extension of the disease process also visible along the contra lateral side with extension into nasal cavity and left sided periorbital tissue. Lesion showing hyper intense signal on T2 iso to low on T1 and post contrast heterogeneous enhancement. 


\section{Discussion}

Sino nasal tumors comprise $3 \%{ }^{1,2}$ of head and neck tumors. Sino nasal tumors are common among men of ages between 55-60 y. ${ }^{3}$ Etiology includes occupational exposure to nickel, wood and leather are common. ${ }^{3}$ Human papilloma virus and Epstein-Barr viruses are also found associated with sino-nasal tumors. ${ }^{4}$ These tumors can be extremely painful and can pose serious psychosocial debilitations.

Different modalities can be used for pain control in maxillofacial tumors, but conventional analgesia is ineffective sometimes and patients may require intervention for pain control. Sphenopalatine ganglion, that innervates lacrimal gland, glands of the nasal cavity, paranasal sinuses, palate, and upper pharynx, can be blocked using different agents such as lignocaine, corticosteroid anesthetic mixture, bupivacaine, alcohol, botulinum toxin $\mathrm{A}$, and cocaine. The SPG plays an important role in controlling various pain syndromes including different type of headaches, trigeminal and sphenopalatine neuralgia, atypical facial pain, muscle pain, vasomotor rhinitis, eye disorders, and post herpetic neuralgia. ${ }^{5}$

A recent study demonstrated the role of SPGB in also relieving post-dural puncture headache (PDPH) syndrome. ${ }^{6}$ Different approaches that can be adopted to block sphenopalatine ganglion include trans nasal, anterior rhinoscope guided, trans-zygomatic with image intensifier. Block of SPG can be achieved either by using drugs like cocaine hydrochloride, alcohol, lignocaine and corticosteroid or by using radiofrequency ablation (RFA) and pulsed radiofrequency (PRF). ${ }^{7}$

Scarcity of literature regarding SPGB in maxillofacial tumors has made our case unique and with promising result as evident as in this case it can be adopted as an effective method to control pain associated with this disease.

\section{Conclusion}

Maxillofacial tumors can be extremely painful and debilitating, often refractory to conventional analgesia. Sphenopalatine ganglion block is a relatively simple procedure, results in significant pain relief in such patients and it must be offered to the patients with painful head and neck tumors.

\section{Conflict of interest}

None declared by the authors

\section{Authors' Contribution}

AU: Conduction of study

FRB: Concept

ARG: Conduction of study work

RSD: Manuscript editing

\section{References}

1. Kalyani R, Narshimhan A, Prakash S. Sinonasal carcinoma-a report of two cases. J Clin Diagn Res. 2014 Feb;8(2):149150. [PubMed] DOI: $10.7860 / J C D R / 2014 / 6848.4037$

2. Lee $\mathrm{CH}$, Hur DG, Roh HJ, Rha KS, Jin HR, Rhee CS, et al. Survival rates of sinonasal squamous cell carcinoma with the new AJCC staging system. Arch Otolaryngol Head Neck Surg. 2007 Feb;133(2):131-134. [PubMed] DOI: 10.1001/archotol.133.2.131

3. Sklar EM, Pizarro JA. Sinonasal intestinal-type adenocarcinoma involvement of the paranasal sinuses. Am J Neuroradiol. 2003 Jun 1;24(6):1152-1155. [PubMed]

4. Leung SY, Yuen ST, Chung LP, Kwong WK, Wong MP, Chan SY. Epstein-Barr virus is present in a wide histological spectrum of sinonasal carcinomas. The Am J Surg Pathol. 1995 Sep;19(9):994-1001. [PubMed] DOI: 10.1097/00000478-199509000-00002

5. Candido KD, Massey ST, Sauer R, Darabad RR, Knezevic NN. A novel revision to the classical transnasal topical sphenopalatine ganglion block for the treatment of headache and facial pain. Pain Physician. 2013 Nov;16:E769-778. [PubMed]

6. Nair AS, Rayani BK. Sphenopalatine ganglion block for relieving postdural puncture headache: technique and mechanism of action of block with a narrative review of efficacy. Korean J Pain. 2017 Apr;30(2):93-97. [PubMed] DOI: 10.3344/kjp.2017.30.2.93

7. Mojica J, Mo B, Ng A. Sphenopalatine ganglion block in the management of chronic headaches. Curr Pain Headache Rep. 2017 Jun 1;21(6):27. [PubMed] DOI: 10.1007/s11916$\underline{017-0626-8}$ 